\title{
Intact neural and behavioral correlates of emotion processing and regulation in weight-recovered anorexia nervosa: a combined fMRI and EMA study
}

\author{
Maria Seidel ${ }^{1}$, Sophie Pauligk ${ }^{1}$, Sophia Fürtjes ${ }^{1}$, Joseph A. King ${ }^{1}$, Sophie-Maleen Schlief ${ }^{1}$, Daniel Geisler ${ }^{1}$, Henrik Walter (iD ${ }^{2}$, \\ Thomas Goschke ${ }^{3}$ and Stefan Ehrlich (D) ${ }^{1,4 凶}$
}

(c) The Author(s) 2022

\begin{abstract}
Altered emotion processing and regulation mechanisms play a key role in eating disorders. We recently reported increased fMRI responses in brain regions involved in emotion processing (amygdala, dorsolateral prefrontal cortex) in acutely underweight anorexia nervosa (AN) patients while passively viewing negatively valenced images. We also showed that patients' ability to downregulate activity elicited by positively valenced pictures in a brain region involved in reward processing (ventral striatum) was predictive of worse outcomes (increased rumination and negative affect). The current study tries to answer the question of whether these alterations are only state effects associated with undernutrition or whether they constitute a trait characteristic of the disorder that persists after recovery. Forty-one individuals that were weight-recovered from AN (recAN) and 41 age-matched healthy controls $(\mathrm{HC})$ completed an established emotion regulation paradigm using negatively and positively valenced visual stimuli. We assessed behavioral (arousal) and fMRI measures (activity in the amygdala, ventral striatum, and dorsolateral prefrontal cortex) during emotion processing and regulation. Additionally, measures of disorder-relevant rumination and affect were collected several times daily for 2 weeks after scanning via ecological momentary assessment. In contrast to our previous findings in acute AN patients, recAN showed no significant alterations either on a behavioral or neural level. Further, there were no associations between fMRI responses and post-scan momentary measures of rumination and affect. Together, these results suggest that neural responses to emotionally valenced stimuli as well as relationships with everyday rumination and affect likely reflect state-related alterations in AN that improve following successful weight-recovery.
\end{abstract}

Translational Psychiatry (2022)12:32; https://doi.org/10.1038/s41398-022-01797-1

\section{INTRODUCTION}

Contemporary models of eating disorders (ED) including anorexia nervosa (AN) propose altered emotion processing and emotion regulation mechanisms to play a key role in the development and maintenance of the disorder [1-4]. Acutely underweight AN patients (acAN) have been reported to have difficulties in general emotional processing [5-8]. In addition, impairments in emotion regulation have also been identified, for example, more frequent use of emotion regulation strategies that are considered maladaptive, such as suppression or rumination $[9,10]$. Most research on emotion processing and regulation in $\mathrm{AN}$ to date has been based on self-report [3]. However, notions of discrepancies between self-reported emotional and physiological reactivity to emotional stimuli [11, 12], as well as high levels of alexithymia in AN [13] warrant a cautious interpretation of these results.

Functional neuroimaging studies generally support the aforementioned behavioral findings suggestive of altered processing of emotions in acAN individuals $[14,15]$ and show differences in neural responses in visual, limbic as well as frontal brain regions [16-19]. We recently reported increased responses of the right amygdala as well as the bilateral dorsolateral prefrontal cortex (dIPFC) in acAN patients while passively viewing negatively valenced images [20]. Results were interpreted as an increased emotional reactivity to negative stimuli (amygdala), possibly as a result of depleted resources due to continued attempts at regulating (increased dIPFC) negative emotions. However, contrary to previous findings from questionnaire and self-report data [3], we did not find any differences between acAN and healthy control participants $(\mathrm{HC})$ in the neural regulation of either negative or positive emotions and corresponding arousal ratings $[20,21]$ during an established task which required emotional reappraisal via distancing [22]. This was found despite the fact that patients generally reported less (trait) reappraisal as assessed with the Emotion Regulation Questionnaire (ERQ [23]. Yet, we also uncovered that patients' ability to downregulate activity in a reward-related brain region (ventral striatum [VS] [24]) during the regulation of positively

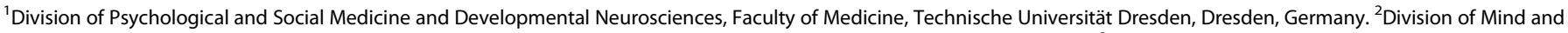
Brain Research, Department of Psychiatry and Psychotherapy CCM, Charité Universitätsmedizin Berlin, Berlin, Germany. ${ }^{3}$ Department of Psychology, Technische Universität

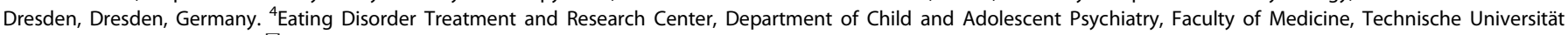
Dresden, Dresden, Germany. ${ }^{凶}$ email: transden.lab@uniklinikum-dresden.de
} 
valenced stimuli was predictive of increased rumination and negative affect, (assessed several times daily via ecological momentary assessment (EMA) for 14 days after the scanning session in real life), and, more importantly, worse treatment outcome (weight gain). Previous research has emphasized the costs and negative consequences of excessive self-control within a limited capacity model $[25,26]$. Consequently, it has been suggested that cognitive processes such as emotion regulation or emotional avoidance might exhaust control resources [21,27], which subsequently has negative consequences (costs) by facilitating the development of ED-related symptoms such as heightened negative affect and ruminative thinking.

Most studies of emotion regulation in $\mathrm{AN}$, including our own previous investigation of neural processing and regulation of emotions [20, 21], focused on acAN and results may thus be partially biased by consequences of insufficient food intake and the cachectic state with all its metabolic and endocrine consequences [28-30]. Therefore, forthcoming research needs to address whether group differences found between acAN and healthy individuals are due to state factors (e.g., the undernourished state) or whether they present a trait marker, potentially influencing the development and maintenance of the disorder. Studying weight-recovered individuals with a history of AN (recAN) could help answer this question. Previous investigations into self-reported aspects of emotion processing and regulation in recAN have yielded heterogeneous results [31-34] while investigations using functional MRI are scarce and have not included an explicit regulation condition [35, 36].

The aim of the current study, therefore, was to investigate possible alterations in the processing of negative emotional stimuli as well as negative consequences of regulating positive emotional stimuli (as previously found in our sample of adolescent acAN) after weight-recovery. To this end, we used the same emotion regulation task $[20,21]$ in recAN to test for persistent alterations in the neural correlates of emotional processing as well as regulation (distancing) as a voluntary strategy to reduce arousal. Using subjective (arousal ratings) and objective (fMRI) data, we were interested not only in whether former patients (recAN) would continue to show increased reactivity to negative stimuli but also display increased negative consequences of regulating positive emotions. As in our previous study in the acAN sample, this was assessed by combining measures of the $\mathrm{fMRI}$ emotion regulation task with data from an EMA study assessing disorder-specific rumination as well as affect in real life.

\section{METHOD}

\section{Participants}

Data were collected from 41 recAN and a total of $50 \mathrm{HC}$. HC were recruited with the goal to match the samples for age. To optimize comparisons between recAN and $\mathrm{HC}$ we implemented a pairwise matching algorithm [37], resulting in a sample of 82 female volunteers: 41 recAN (15.4-29 years) and 41 female HCs (15.5-29.7 years). This procedure resulted in a maximum difference of 0.8 years between matched pairs. There was no overlap in participants between the acAN patients in our previous studies $[20,21]$ and the current sample of weight-recovered participants. All recAN participants had an AN diagnosis in the past according to DSM-IV obtained with the expert version of the Structured Interview for anorexia and bulimia nervosa (SIAB-EX [38]). To be considered "weight-recovered", recAN subjects had to (1) maintain a $\mathrm{BMI}\left(\mathrm{kg} / \mathrm{m}^{2}\right)>18.5$ (if older than 18 years) or above the 10th age percentile (if younger than 18 years) for at least 6 months; (2) menstruate; and (3) have not binged, purged, or engaged in restrictive eating patterns during at least 6 months before the study. Further sample descriptives are provided in Table 1. HC participants had to be of normal weight, eumenorrhoeic, and without any history of psychiatric disorder. Exclusion criteria for both groups and possible confounding variables, e.g., the use of psychotropic medication and medical comorbidities, were obtained using the SIAB-EX [38], our own semi-structured research interview, and from medical records. For additional exclusion criteria see supplementary material 1.1.

This study was approved by the local institutional ethics review board, and all participants (and their legal guardians if underage) gave written informed consent.

\section{Clinical measures}

To complement the information obtained with the clinical interviews, we assessed ED-specific psychopathology using the Eating Disorder Inventory (EDI-2) [39] and depressive symptoms using the Beck Depression Inventory (BDI-II) [40]. For trait use of the emotion regulation strategies reappraisal and suppression, we used the Emotion Regulation Questionnaire (ERQ) [41]. BMI and BMI standard deviation scores corrected for gender and age (BMI-SDS) [42] were collected on the day of scanning.

\section{Emotion regulation task}

During the task, participants were asked to either passively view sets of negative, positive, and neutral pictures or to actively downregulate any emotions arising in response to the negative and positive pictures $[43,44]$. During the "view" condition participants were instructed to simply look at the picture without modulating any associated feelings, without looking away or distracting themselves in any way. During the regulation condition, they were told to downregulate any elicited feeling via the reappraisal strategy "distancing". After each picture presentation (6s) participants were asked to rate their emotional arousal. Participants first completed a practice session. The main task consisted of 100 trials (20 per condition) which were presented in pseudorandomized order with each

Table 1. Descriptive statistics, results of group comparisons using independent samples $T$-tests, displaying mean and standard deviation.

\begin{tabular}{|c|c|c|c|c|c|c|}
\hline \multirow[t]{2}{*}{ Descriptive Statistics } & \multirow[t]{2}{*}{ (recAN/HC) } & \multicolumn{2}{|l|}{ recAN } & \multicolumn{3}{|l|}{ HC } \\
\hline & & Mean & SD & Mean & SD & \\
\hline Age & $41 / 41$ & 22.10 & 3.67 & 22.05 & 3.68 & \\
\hline BMI & $41 / 41$ & 20.65 & 1.60 & 21.93 & 2.02 & ** \\
\hline BMI-SDS & $41 / 41$ & -0.53 & 0.56 & -0.11 & 0.56 & ** \\
\hline Duration of Recovery & $41 / 41$ & 58.72 & 54.97 & & & \\
\hline BDI-II & $41 / 41$ & 10.15 & 10.00 & 4.40 & 4.66 & * \\
\hline EDI-total & $41 / 40$ & 178.93 & 52.36 & 139.29 & 25.00 & * \\
\hline ERQ-Reappraisal & $41 / 41$ & 48.20 & 8.99 & 50.98 & 10.12 & \\
\hline ERQ-Suppression ${ }^{\mathrm{a}}$ & $41 / 41$ & 51.07 & 9.24 & 49.17 & 8.70 & \\
\hline
\end{tabular}

Duration of recovery is given in months, range: $9-270$ months (only $n=2<12$ months). Previous AN diagnoses of recovered individuals included $n=34$ of the restrictive subtype and $n=8$ of the binge/purge subtype.

RecAN recovered Anorexia nervosa, $H C$ healthy control, BMI body-mass index, BMI-SDS BMI standard deviation score, EDI-2-total eating disorder inventory 2 total score, $B D I-I /$ Beck depression inventory, SD standard deviation.

${ }^{* * *} p<0.001,{ }^{* *} p<0.01,{ }^{*} p<0.05$.

${ }^{a}$ As normal distribution of variables was not given group comparisons were repeated with Mann-Whitney U-tests. Results did not change (supplementary material Table S1). 
condition constrained to not occur more than twice in a row, while the assignment of stimuli to either the "view" or "distance" condition was randomized for each participant. The fMRI measurement lasted for 23 min. See supplementary material 1.2 or Seidel et al. [20, 21] for more details about the task and procedure.

\section{Ecological momentary assessment}

Rumination about AN-related content (food/weight) was assessed via two items adapted from the SIAB-EX interview i.e., "How much have you been thinking about food/calories/cooking?" and "How much have you been thinking about your weight/shape?". Responses were ranging from "not at all" to "a lot".

An adapted version of the Multidimensional Mood Questionnaire (MDMQ) [45] recommended to use in EMA research [46] assessed calmness and valence of affect with two bipolar items each. Higher scores indicated more positive affect and more calmness.

The app-based questionnaire was designed via an online platform (MovisensXS, Karlsruhe, Germany), which also managed data collection and immediate server upload. EMA sampling started the day after the fMRI scan and lasted for a period of 14 days. Data collection occurred via the signal-contingent assessment method: Alarms occurred at six semirandom times during a $14 \mathrm{~h}$ period that was adapted for each individual to suit different daily routines. For further details of the EMA study design and procedure see Seidel et al. [10] or supplementary material 1.3.

\section{Functional image acquisition and processing}

Images were acquired between 8 and 9 a.m. following an overnight fast using standard sequences with a $3 \mathrm{~T}$ whole-body MRI scanner (TRIO, Siemens) equipped with a standard head coil. Functional and structural images were processed with SPM8 (www.fil.ion.ucl.ac.uk/spm) within the Nipype framework (http://nipy.sourceforge.net/nipype/37) following standard procedures, an artifact detection tool and DARTEL (for details of fMRI acquisition and processing, see supplementary material 1.4).

On the single participant level a general linear model (GLM) was fit to model the brain activation in response to each of the five conditions (neutral; positive watch or distance; negative watch or distance). We modeled the picture as a boxcar function with a duration of $6 \mathrm{~s}$ and the subsequent rating as stick-function (zero duration). Additional regressors included six motion parameters and one regressor for each motion or intensity outlier volume as nuisance regressors of no interest. All events were modeled using a canonical hemodynamic response function.

\section{Statistical analysis}

Analysis strategies for task-related effects of negative and positive emotional processing and regulation, as well as EMA data were based on our previous analyses in an independent sample of acAN as outlined in the introduction [20, 21]. Therefore, primary analyses comprised all analyses including variables that were found to be different between acAN and HC in our previous studies [20,21]. Secondary analyses included group differences in the main effects of tasks and associations with clinical and demographic variables.

Clinical and behavioral task-based data. Analyses included independent samples $t$-tests or Mann-Whitney $U$-tests (if the normal distribution was not given), to compare clinical and questionnaire data between groups using SPSS 26 . Secondary analyses were comprised of a $5 \times 2$ repeated measures ANOVA, to test for potential group differences across the conditions of the emotion regulation task (neutral, negative watch, negative distance, positive watch, and positive distance) in the arousal ratings.

Functional MRI. The first part of the primary analysis included the investigation of group differences in the contrast negative watch $>$ neutral (as in acAN [20]). Regions of interests (ROIs) were defined by AAL regions with significant group differences between acAN and $\mathrm{HC}$ in this contrast, i.e., right amygdala and right and left dIPFC, as implemented in the WFU PickAtlas toolbox for SPM [47, 48]. Of note, as no significant group differences had been found in the left amygdala all contrasts for the ROI were investigated as part of the secondary analysis, see below. To calculate group differences between recAN and $\mathrm{HC}$ we applied second level independent samples $t$-tests within the respective ROI. To control for false positives, familywise error correction was performed using 3DClustSim (https://afni.nimh.nih.gov/, version from 3 July 2017). Specifically, the program was used to run 10,000 Monte Carlo simulations to estimate the cluster size above which the false positive probability is below a given alevel $(a=0.05)$ for a given voxel-wise $p$ value, which was set at 0.001 . At this voxel-wise threshold (two-sided), clusters with more than six voxels for the right amygdala and 33 voxels for the left and right dIPFC each, corresponding to a combined threshold of $p<.05$ [familywise error (FWE) corrected]. In case of nonsignificant group differences in the data derived from neuroimaging, we used the JASP software to calculate Bayesian independent $t$-tests to verify the absence of any group effects [49]. In order to do so, we averaged indices of activation ( $\beta$ estimates) which were extracted from the aforementioned ROls using the MarsBaR toolbox [50]. A Bayes factor (BF01) $>3$ represents moderate and BF01 $>10$ strong evidence for the null hypotheses (no group differences).

The second part of the primary analysis investigated group differences in the association between neural indices of positive emotion regulation with post-scan measures of affect and rumination in real life (EMA data; as found in acAN [21]). Mirroring the analyses strategy in our acAN study, we calculated a positive neural emotion regulation score using extracted betas from the VS (defined by AAL regions in which we had found significant group differences between acAN and $\mathrm{HC}$ ). The score was calculated by subtracting the extracted betas within the VS during the condition positive distance from the positive watch. Subsequently, the higher the positive neural regulation score, the more neural activity was reduced during the regulation condition as compared to the watch condition. For further analyses investigating associations between the positive neural regulation score and EMA data refer to the hierarchical linear model section.

Secondary analyses included investigating group differences during watching negative pictures (negative watch>neutral (left amygdala)), regulation of negative pictures (contrasts: negative watch<negative distance (bilateral amygdala), negative watch $>$ negative distance (bilateral dIPFC)) as well as viewing and regulation of positive pictures (positive watch $>$ neutral (VS), positive watch $>$ positive distance (VS), positive watch<positive distance (bilateral dIPFC)) via independent $t$-tests. Again, clusters with more than six voxels for the right/left amygdala and 33 voxels for the left and right dIPFC each, corresponding to a combined threshold of $p<.05$ [familywise error (FWE) corrected. Secondary analyses also included whole-brain analyses as well as investigating associations between imaging and behavioral and clinical data (supplementary material 1.5.1).

Hierarchical linear models. As not all participants had provided EMA data, HLM analyses were calculated on a subsample of 65 (recAN $=30, \mathrm{HC}=35$ ) individuals. EMA data of affective variables and rumination of a largely overlapping $(70.73 \%)$ sample of weight-recovered patients has been published previously [51]. As the research design of the EMA data yields nested data, primary analyses included conducting hierarchical linear models (HLM 8) [52]. As in our previous study we set up four different models to examine the extent to which positive neural regulation was able to predict disorder-relevant rumination (food: model a; weight: model b), the valence of effect (model c), and calmness (model d) in the 14 days following the scan [21]. These models took into account that the dataset was organized within three different levels with single observations (Level 1) nested within days (Level 2) which were nested within participants (Level 3). The same statistical approach was used for all models. In model a-d we allowed for random intercepts and included time (indicating time of day as a continuous variable from 1 to 6 ) on level 1 and day of study (1 to 14) on level 2. On level 3, the person level, we included three predictors. The first diagnostic group was inserted, coded as 1 (recAN) and $-1(\mathrm{HC})$. Second, we entered the mean-centered positive neural regulation score (positive watch - positive distance) of the extracted betas from the VS (as described above), and thirdly we included an interaction term of this regulation score with a diagnostic group. For supplementary analysis we also looked at effects of negative emotion regulation on rumination and negative affect by conducting separate HLMs that included extracted data from the amygdala during negative regulation as a predictor instead of the VS (for details see supplementary material 1.5.2).

\section{RESULTS}

\section{Clinical variables and behavioral task-based data}

We did not find any differences between recAN and $\mathrm{HC}$ in age or $I Q$, but BMI was significantly lower and ED symptoms (EDI-2), as well as depression scores (BDI-II), were still elevated (Table 1). As indicated by ERQ subscale scores, recAN did not show any 
differences in the general use of the emotion regulation strategies reappraisal and suppression. As normal distribution was not given for BDI-II and ERQ-suppression scales, group comparisons were repeated using Mann-Whitney $U$-tests. The significance of the results was identical (supplementary material Table S1). Secondary analyses of arousal ratings acquired during the fMRI task revealed no group differences in the five conditions (see supplementary material 2.1).

\section{Functional MRI}

Contrary to our previous findings in acAN [20], primary analyses revealed no group differences in neural processing of negative stimuli in the right amygdala and the bilateral dIPFC between recAN and $\mathrm{HC}$ (contrast: negative watch>neutral). Post hoc analysis using Bayesian independent samples $t$-tests showed moderate evidence in favor of the null hypothesis $\left(\mathrm{BF}_{01}\right.$ right Amygdala $=4.19, \mathrm{BF}_{01}$ right dIPFC $=4.32, \mathrm{BF}_{01}$ left dIPFC $=4.32$ ) confirming the absence of group differences between recAN and $\mathrm{HC}$ in these ROls.

Results of secondary analyses investigating group differences in effects of the task as well as analysis of associations between neuroimaging and questionnaire data are presented in the supplementary material 2.2 (fMRl task-based findings) and 2.3 (associations between behavioral and neuroimaging findings). In short, there were no group differences in the neural activity in any of the contrasts in any ROI (negative watch>neutral left amygdala, watch $>$ negative distance in the bilateral amygdala and positive watch>neutral, positive watch>positive distance in the VS and positive watch<positive distance in the bilateral dIPFC). The downregulation of amygdala activity in the negative watch>negative distance contrast was mirrored by the downregulation of subjective arousal (supplementary Table S2).

\section{Hierarchical linear models for EMA data}

Applying the same analytic approach previously employed in our acAN sample [21], we tested the effect of the positive neural regulation score based on VS activity (and its interaction with the group factor) on rumination about weight (model a), rumination about food (model b), affect (model c), and calmness (model d) based on a total of 4361 data points.

The recAN group showed elevated rumination about weight, more negative affect, and less calmness than HC (for more details on EMA data see supplementary material Table S3 or Fürtjes et al. [51]). However, contrary to our previous findings in acAN, there was neither the main effect of positive neural regulation nor a significant positive neural regulation $x$ group interaction in any of the models. Evidently, neural downregulation of the VS did not predict subsequent rumination about weight, rumination about food, negative affect, or calmness levels in either group (see supplementary material Table S3. Supplementary analysis investigating effects of negative emotion regulation on EMA data did not show any significant effects (supplementary Tables S4 and S5).

\section{DISCUSSION}

Building on our previous findings in acAN patients [20, 21], the current analysis sought to test for persistent alterations in the processing of negatively valenced emotional stimuli and the reallife consequences of regulation of positive emotions after weightrecovery from AN. In contrast to our findings in acAN [20], primary analyses revealed no group differences between recAN and $\mathrm{HC}$ in the amygdala and dIPFC during passive viewing of negatively valenced images. Further, there was no significant association between the voluntary downregulation of neural activity in the VS in response to positively valenced stimuli and either negative effect or disorder-related rumination in real life during the 2 weeks after the scanning session (although negative affect and rumination were still elevated in recAN). In line with that, questionnaire data confirmed that recAN use trait reappraisal to the same degree as $\mathrm{HC}$, suggestive of a normalization process after recovery. Secondary analyses showed that recAN also did not display any difficulties downregulating negative and positive emotions when instructed to use distancing, as measured using arousal ratings and neural activity in the amygdala, the VS, as well as dIPFC. When considered in light of our previous findings in acAN, the data at hand suggest that alterations of emotional processing and increased negative real-life consequences of regulation do not persist following long-term weight-recovery and are thus more likely to constitute correlates of the underweight state than trait factors of the disorder.

In contrast to the increased amygdala and dIPFC activation during passive viewing of negative stimuli we previously observed in acAN [20], we found no evidence of any such neural alterations in recAN. The amygdala is a complex structure important for detecting potentially threatening and fearful stimuli [53]. As such, it is highly responsive towards all emotionally valenced stimuli, particularly emotional faces [54, 55]. Functional alterations of the amygdalae appear to characterize especially anxiety-prone [56] or depressed individuals $[57,58]$, characteristics also displayed by individuals suffering from acute AN [59-61]. As general anxiety levels and negative affect decrease during recovery $[62,63]$ they might be partly responsible for the fact that the amygdala does not display exaggerated reactivity towards negative emotional stimuli in recAN. Although we did not observe a direct association between depressive or anxiety symptoms and amygdala activity in acAN [20], reported symptoms were only based on self-report data and more objective measures might yield different results [64].

PFC areas have been shown to exert top-down regulatory control over emotion modulating limbic brain regions (such as the amygdala) in order to promote successful emotion regulation [65]. We, therefore, suggested that increased dIPFC activity in acAN may reflect a compensatory response to elevated amygdala reactivity. However, based on assumptions of limited control capacities [66,67], the opposite could also have been the case. The observed dIPFC hyperactivity could have depleted resources which in turn led to increased vulnerability of the amygdala and increased activity to negative stimuli $[68,69]$. This latter interpretation was further supported by our follow-up study [27] that found increased dIPFC activity in acAN patients during the emotion regulation task predicted subsequent higher amygdala response to negative and neutral stimuli that were presented shortly after the main experiment. Given that it might no longer be necessary to constantly regulate (e.g., feelings of hunger and bodily signals promoting eating [62]) after weight-recovery, a speculative interpretation of our current findings in recAN could be that control mechanisms exerted by the dIPFC are only active when needed. Hence, resources needed for regulation (e.g., in the event of negative emotions) might be more readily available following recovery and therefore would not promote increased vulnerability or amygdala reactivity. Our result indicative of equivalent neural activation between recAN and $\mathrm{HC}$ is consistent with work on other emotional disorder-unrelated stimuli, which found intact neural processing of emotional stimuli in recAN $[36,70]$. However, the results deviate from other neuroimaging studies in recAN that show blunted amygdala activity in response to disorder-unrelated (facial) stimuli [71] or have employed disorder-related stimuli such as food pictures [72-74], taste [75-78], or body stimuli [79].

Although no neural differences in processing and regulation of positive stimuli were observed in our previous study of acAN [21], we did find those patients, who were better able to downregulate neural activity in the VS, were also the ones suffering from worse consequences in everyday life in terms of negative affect, more tension, increased disorder-related rumination and even less weight gain after 60 and 90 days of specialized treatment. Drawing on theories of limited cognitive resources [66], we 
interpreted worse short- and long-term outcomes as real-life costs of control in terms of possible consequences of maladaptive control tendencies that are common in acAN [29] and an emerging therapeutic target [80, 81]. Relatedly, ironic processing theory $[82,83]$ states that suppressed thought actually becomes more accessible and backfire with intrusive thoughts (e.g., disorder-related rumination), increased risk of depression, and negative affect. Based on these interpretations, we speculate that control tendencies are either not as strong or not as costly for recAN compared to during the acutely underweight state. This apparent reduction of overcontrol in recAN dovetails with recent findings from our group suggesting that the neural mechanisms underlying self-controlled choice normalize over the course of recovery $[70,84]$. Alternatively, it is possible that tendencies to avoid (e.g., suppress) emotions or specific thought content decrease in recAN [34], allowing for more adaptive processing of emotional as well as eating disorder-related content [62] with less intrusive thoughts.

Our findings of similar emotion regulation processes in recAN and $\mathrm{HC}$ are in line with questionnaire data reported who also found no differences in self-reported emotion regulation capacities of remitted AN individuals [34, 85]. However, others reported that difficulties with emotion regulation as assessed via self-report persist even after recovery [31], highlighting the importance of applying measures that go beyond questionnaire data.

The following limitations should be considered when interpreting the results of this study: Given the systematic age differences between acAN from our previous manuscript and the weightrecovered sample, we abstained from comparing all three groups in one statistical model which may have delivered different results. Our study was intentionally designed in this manner with the purpose of conducting separate (but nonetheless identical) analyses addressing state vs. trait effects. To clarify the question of normalization of emotional processes during recovery, beyond correlational analysis, future research applying longitudinal study designs is strongly encouraged. Secondly, although we gave explicit instructions including pre-scan training, we cannot be absolutely sure which emotion regulation strategy participants applied during the task. Lastly, in order to avoid anxious emotional states, we only investigated emotion regulation to a stimulus set, which was strictly filtered regarding ED-related content, and therefore our findings may not generalize to disorder-relevant stimulus content [86].

Our previously observed alterations in neural processing of negative emotions, as well as associations between emotion regulation of positive stimuli and outcome measures in acAN $[20,21])$ were not visible in recAN individuals. Thus, the current findings provide evidence for a relative normalization following weight-recovery, suggesting that aberrant neural mechanisms underlying emotion processing and regulation might represent state factors associated with undernutrition rather than a disorderdefining trait. Nevertheless, the extent and speed of normalization over the course of recovery remains an open question. Overall, our results may send a positive message to patients, while also being useful information for patient education and therapeutic interventions $[81,87,88]$.

\section{REFERENCES}

1. Lavender JM, Wonderlich SA, Engel SG, Gordon KH, Kaye WH, Mitchell JE. Dimensions of emotion dysregulation in anorexia nervosa and bulimia nervosa: a conceptual review of the empirical literature. Clin Psychol Rev. 2015;40:111-22.

2. Oldershaw A, Startup $H$, Lavender T. Anorexia nervosa and a lost emotional self: a psychological formulation of the development, maintenance, and treatment of anorexia nervosa. Front Psychol. 2019;10:219.

3. Oldershaw A, Lavender T, Sallis H, Stahl D, Schmidt U. Emotion generation and regulation in anorexia nervosa: a systematic review and meta-analysis of selfreport data. Clin Psychol Rev. 2015;39:83-95.
4. Prefit A-B, Cândea DM, Szentagotai-Tătar A. Emotion regulation across eating pathology: a meta-analysis. Appetite. 2019;143:104438.

5. Brockmeyer T, Bents H, Holtforth MG, Pfeiffer N, Herzog W, Friederich H-C. Specific emotion regulation impairments in major depression and anorexia nervosa. Psychiatry Res. 2012;200:550-3.

6. Harrison A, Sullivan S, Tchanturia K, Treasure J. Emotion recognition and regulation in anorexia nervosa. Clin Psychol Psychother. 2009;16:348-56.

7. Kolar DR, Huss M, Preuss HM, Jenetzky E, Haynos AF, Bürger A, et al. Momentary emotion identification in female adolescents with and without anorexia nervosa. Psychiatry Res. 2017;255:394-8.

8. Parling T, Mortazavi M, Ghaderi A. Alexithymia and emotional awareness in anorexia nervosa: time for a shift in the measurement of the concept? Eat Behav. 2010;11:205-10

9. Aldao A, Nolen-Hoeksema S, Schweizer S. Emotion-regulation strategies across psychopathology: a meta-analytic review. Clin Psychol Rev. 2010;30:217-37.

10. Seidel M, Petermann J, Diestel S, Ritschel F, Boehm I, King JA, et al. A naturalistic examination of negative affect and disorder-related rumination in anorexia nervosa. Eur Child Adolesc Psychiatry. 2016;25:1207-16.

11. Zonnevylle-Bender MJ, van Goozen SH, Cohen-Kettenis PT, Jansen LM, van Elburg $A$, van Engeland $\mathrm{H}$. Adolescent anorexia nervosa patients have a discrepancy between neurophysiological responses and self-reported emotional arousal to psychosocial stress. Psychiatry Res. 2005;135:45-52.

12. Nandrino J-L, Berna G, Hot P, Dodin V, Latrée J, Decharles S, et al. Cognitive and physiological dissociations in response to emotional pictures in patients with anorexia. J Psychosom Res. 2012;72:58-64.

13. Westwood H, Kerr-Gaffney J, Stahl D, Tchanturia K. Alexithymia in eating disorders: systematic review and meta-analyses of studies using the Toronto Alexithymia Scale. J Psychosom Res. 2017;99:66-81.

14. Olivo G, Gaudio S, Schiöth HB (2019): Brain and cognitive development in adolescents with anorexia nervosa: a systematic review of fMRI studies. Nutrients. 2019;11:1907.

15. Zhu Y, Hu X, Wang J, Chen J, Guo Q, Li C, et al. Processing of food, body and emotional stimuli in anorexia nervosa: a systematic review and meta-analysis of functional magnetic resonance imaging studies. Eur Eat Disord Rev J Eat Disord Assoc. 2012;20:439-50.

16. Fonville L, Giampietro V, Surguladze S, Williams S, Tchanturia K. Increased BOLD signal in the fusiform gyrus during implicit emotion processing in anorexia nervosa. Neurolmage Clin. 2014;4:266-73.

17. Horndasch S, Roesch J, Forster C, Dörfler A, Lindsiepe S, Heinrich H, et al. Neural processing of food and emotional stimuli in adolescent and adult anorexia nervosa patients. PLoS ONE. 2018;13:e0191059.

18. Lulé $D$, Müller S, Fladung A-K, Uttner I, Schulze UM. Neural substrates of anorexia nervosa patient's deficits to decode emotional information. Eat Weight Disord.2020;26:723-8.

19. Steward T, Martínez-Zalacaín I, Mestre-Bach G, Sánchez I, Riesco N, JiménezMurcia $\mathrm{S}$, et al. Dorsolateral prefrontal cortex and amygdala function during cognitive reappraisal predicts weight restoration and emotion regulation impairment in anorexia nervosa. Psychol Med. 2020;1-9.

20. Seidel M, King JA, Ritschel F, Boehm I, Geisler D, Bernardoni F, et al. Processing and regulation of negative emotions in anorexia nervosa: an fMRI study. Neurolmage Clin. 2018;18:1-8.

21. Seidel M, King JA, Ritschel F, Boehm I, Geisler D, Bernardoni F, et al. The real-life costs of emotion regulation in anorexia nervosa: a combined ecological momentary assessment and fMRI study. Transl Psychiatry. 2018;8:28.

22. Ochsner KN, Bunge SA, Gross JJ, Gabrieli JDE. Rethinking feelings: an fMRI study of the cognitive regulation of emotion. J Cogn Neurosci. 2002;14:1215-29.

23. Gratz KL, Roemer L. Multidimensional assessment of emotion regulation and dysregulation: development, factor structure, and initial validation of the difficulties in emotion regulation scale. J Psychopathol Behav Assess. 2004;26:41-54.

24. Staudinger MR, Erk S, Walter $\mathrm{H}$. Dorsolateral prefrontal cortex modulates striatal reward encoding during reappraisal of reward anticipation. Cereb Cortex. 2011;21:2578-88

25. Baumeister RF. Ego depletion and self-regulation failure: a resource model of selfcontrol. Alcohol Clin Exp Res. 2003;27:281-4.

26. Garrison KE, Finley AJ, Schmeichel BJ (2019): Ego depletion reduces attention control: evidence from two high-powered preregistered experiments. Pers Soc Psychol Bull. 2019;45:728-39.

27. Pauligk S, Seidel M, Fürtjes S, King JA, Geisler D, Hellerhoff I, et al. The costs of over-control in anorexia nervosa: evidence from $\mathrm{fMRI}$ and ecological momentary assessment. Transl Psychiatry. 2021;11:304.

28. Frank GKW. Altered brain reward circuits in eating disorders: chicken or egg? Curr Psychiatry Rep. 2013;15:396.

29. Kaye WH, Wierenga CE, Bailer UF, Simmons AN, Bischoff-Grethe A. Nothing tastes as good as skinny feels: the neurobiology of anorexia nervosa. Trends Neurosci. 2013;36:110-20. 
30. Støving RK. MECHANISMS IN ENDOCRINOLOGY: anorexia nervosa and endocrinology: a clinical update. Eur J Endocrinol. 2019;180:R9-27.

31. Brockmeyer T, Holtforth MG, Bents H, Kämmerer A, Herzog W, Friederich H-C. Starvation and emotion regulation in anorexia nervosa. Compr Psychiatry. 2012;53:496-501.

32. Brown TA, Cusack A, Berner LA, Anderson LK, Nakamura T, Gomez L, et al. Emotion regulation difficulties during and after partial hospitalization treatment across eating disorders. Behav Ther. 2020;51:401-12.

33. Haynos AF, Roberto CA, Martinez MA, Attia E, Fruzzetti AE. Emotion regulation difficulties in anorexia nervosa before and after inpatient weight restoration. Int J Eat Disord.2014;47:888-91.

34. Oldershaw A, DeJong H, Hambrook D, Broadbent H, Tchanturia K, Treasure J, et al. Emotional processing following recovery from anorexia nervosa. Eur Eat Disord Rev. 2012;20:502-9.

35. Bang L, Rø Ø, Endestad T. Amygdala alterations during an emotional conflict task in women recovered from anorexia nervosa. Psychiatry Res Neuroimaging. 2016:248:126-33.

36. Cowdrey FA, Harmer CJ, Park RJ, McCabe C. Neural responses to emotional faces in women recovered from anorexia nervosa. Psychiatry Res. 2012;201:190-5.

37. Munkres J. Algorithms for the assignment and transportation problems. J Soc Ind Appl Math. 1957;5:32-8.

38. Fichter M, Quadflieg N. The structured interview for anorexic and bulimic disorders for DSM-IV and ICD-10 (SIAB-EX): reliability and validity. Eur Psychiatry J Assoc Eur Psychiatr. 2001;16:38-48.

39. Thiel A, Jacobi C, Horstmann S, Paul T, Nutzinger DO, Schüßler G. Eine deutschsprachige Version des Eating Disorder Inventory EDI-2. [German translation of the Eating Disorder Inventory EDI-2.]. Psychother Psychosom Med Psychol. 1997;47:365-76.

40. Hautzinger M, Keller F, Kuehner, C. Beck Depression Inventory II (BDI-II), German Version [in German]. Harcourt Test Services: Frankfurt a. M; 2006.

41. Gross JJ, John OP. Individual differences in two emotion regulation processes: Implications for affect, relationships, and well-being. J Pers Soc Psychol. 2003;85:348-62.

42. Kromeyer-Hauschild K, Wabitsch M, Kunze D, Geller F, Geiß HC, Hesse V, et al. Perzentile für den body-mass-index für das Kindes- und Jugendalter unter Heranziehung verschiedener deutscher Stichproben. Monatsschr Kinderheilkd. 2001;149:807-18.

43. Lang $P$, Bradley $M$, Cuthbert B. International affective picture system (IAPS): affective ratings of pictures and instruction manual. Gainesville (FL): University of Florida; 2008

44. Wessa M, Kanske P, Neumeister P, Bode K, Heissler J, Schönfelder S. EmoPicS: subjektive und psychophysiologische evaluation neuen bildmaterials für die klinisch-biopsychologische Forschung. 2010

45. Steyer R, Schwenkmezger P, Notz P, Eid M. Der mehrdimensionale Befindlichkeitsfragebogen (MDBF). handanweisung [The multidimensional affect rating scale (MDBF). Manual]. Göttingen, Germany: Hogrefe; 1997.

46. Wilhelm P, Schoebi D. Assessing mood in daily life: structural validity, sensitivity to change, and reliability of a short-scale to measure three basic dimensions of mood. Eur J Psychol Assess. 2007;23:258-67.

47. Maldjian JA, Laurienti PJ, Kraft RA, Burdette JH. An automated method for neuroanatomic and cytoarchitectonic atlas-based interrogation of fMRI data sets. Neurolmage. 2003;19:1233-9.

48. Tzourio-Mazoyer N, Landeau B, Papathanassiou D, Crivello F, Etard O, Delcroix N, et al. Automated anatomical labeling of activations in SPM using a macroscopic anatomical parcellation of the MNI MRI single-subject brain. Neurolmage. 2002;15:273-89.

49. Keysers C, Gazzola V, Wagenmakers E-J. Using Bayes factor hypothesis testing in neuroscience to establish evidence of absence. Nat Neurosci. 2020;23:788-99.

50. Brett $\mathrm{M}$, Anton J-L, Valabregue R, Poline J-B. Region of interest analysis using the MarsBar toolbox for SPM 99. Neuroimage. 2002;16:S497.

51. Fürtjes $S$, Seidel $M$, King JA, Boehm I, Jaite $C$, Roessner $V$, et al. A naturalistic investigation of cognitive-affective dysfunction in anorexia nervosa: the role of inefficiency. Int J Eat Disord. 2019;53:239-47

52. Raudenbush SW, Bryk AS. Hierarchical linear models: applications and data analysis methods. Sage; 2002.

53. Öhman A. The role of the amygdala in human fear: automatic detection of threat. Psychoneuroendocrinology. 2005;30:953-8.

54. Adolphs R. Fear, faces, and the human amygdala. Curr Opin Neurobiol. 2008; 18:166-72.

55. Sergerie K, Chochol C, Armony JL. The role of the amygdala in emotional processing: a quantitative meta-analysis of functional neuroimaging studies. Neurosci Biobehav Rev. 2008;32:811-30.

56. Stein MB, Simmons AN, Feinstein JS, Paulus MP. Increased amygdala and insula activation during emotion processing in anxiety-prone subjects. Am J Psychiatry. 2007;164:318-27.
57. Rakesh D, Allen NB, Whittle S. Balancing act: Neural correlates of affect dysregulation in youth depression and substance use - A systematic review of functional neuroimaging studies. Dev Cogn Neurosci. 2020;42:100775.

58. Victor TA, Furey ML, Fromm SJ, Öhman A, Drevets WC. Relationship between amygdala responses to masked faces and mood state and treatment in major depressive disorder. Arch Gen Psychiatry. 2010;67:1128-38.

59. Bühren K, Schwarte R, Fluck F, Timmesfeld N, Krei M, Egberts K, et al. Comorbid psychiatric disorders in female adolescents with first-onset anorexia nervosa. Eur Eat Disord Rev. 2014;22:39-44.

60. Carrot B, Radon L, Hubert T, Vibert S, Duclos J, Curt F. et al. Are lifetime affective disorders predictive of long-term outcome in severe adolescent anorexia nervosa? Eur Child Adolesc Psychiatry. 2017;26:969-78.

61. Kaye WH, Bulik CM, Thornton L, Barbarich N, Masters K, the Price Foundation Collaborative Group. Comorbidity of anxiety disorders with anorexia and bulimia nervosa. Am J Psychiatry. 2004;161:2215-21.

62. Fürtjes S, Seidel M, King JA, Biemann R, Roessner V, Ehrlich S. Rumination in anorexia nervosa: cognitive-affective and neuroendocrinological aspects. Behav Res Ther. 2018;111:92-8.

63. Young KS, Rennalls SJ, Leppanen J, Mataix-Cols D, Simmons A, Suda M, et al. Exposure to food in anorexia nervosa and brain correlates of food-related anxiety: findings from a pilot study. J Affect Disord. 2020;274:1068-75.

64. Salbach-Andrae H, Klinkowski N, Lenz K, Pfeiffer E, Lehmkuhl U, Ehrlich S. Correspondence between self-reported and parent-reported psychopathology in adolescents with eating disorders. Psychopathology. 2008;41:307-12.

65. Ochsner KN, Silvers JA, Buhle JT. Functional imaging studies of emotion regulation: a synthetic review and evolving model of the cognitive control of emotion. Ann N. Y Acad Sci. 2012;1251:E1-24.

66. Muraven M, Tice DM, Baumeister RF. Self-control as a limited resource: regulatory depletion patterns. J Pers Soc Psychol. 1998;74:774-89.

67. Vohs KD, Heatherton TF. Self-regulatory failure: a resource-depletion approach. Psychol Sci. 2000;11:249-54.

68. Wagner DD, Altman M, Boswell RG, Kelley WM, Heatherton TF. Self-regulatory depletion enhances neural responses to rewards and impairs top-down control. Psychol Sci. 2013;24:2262-71.

69. Polivy J. Psychological consequences of food restriction. J Am Diet Assoc. 1996;96:589-92.

70. King JA, Bernardoni F, Geisler D, Ritschel F, Doose A, Pauligk S, et al. Intact valuebased decision-making during intertemporal choice in women with remitted anorexia nervosa? An fMRI study. J Psychiatry Neurosci. 2019;44:180252.

71. Leppanen J, Cardi V, Paloyelis Y, Simmons A, Tchanturia K, Treasure J. Blunted neural response to implicit negative facial affect in anorexia nervosa. Biol Psychol. 2017;128:105-11.

72. Holsen LM, Lawson EA, Christensen K, Klibanski A, Goldstein JM. Abnormal relationships between the neural response to high- and low-calorie foods and endogenous acylated ghrelin in women with active and weight-recovered anorexia nervosa. Psychiatry Res Neuroimaging. 2014;223:94-103.

73. Oberndorfer T, Simmons A, McCurdy D, Strigo I, Matthews S, Yang T, et al. Greater anterior insula activation during anticipation of food images in women recovered from anorexia nervosa versus controls. Psychiatry Res Neuroimaging. 2013;214:132-141.

74. Uher R, Brammer MJ, Murphy T, Campbell IC, Ng VW, Williams SC, et al. Recovery and chronicity in anorexia nervosa: brain activity associated with differential outcomes. Biol Psychiatry. 2003;54:934-42.

75. Cowdrey FA, Park RJ, Harmer CJ, McCabe C. Increased neural processing of rewarding and aversive food stimuli in recovered anorexia nervosa. Biol Psychiatry. 2011;70:736-43.

76. Kaye WH, Wierenga $C E$, Bischoff-Grethe $A$, Berner LA, Ely AV, Bailer UF, et al. Neural insensitivity to the effects of hunger in women remitted from anorexia nervosa. Am J Psychiatry. 2020;177:601-10.

77. Oberndorfer T, Frank GKW, Simmons AN, Wagner A, McCurdy D, Fudge JL, et al. Altered insula response to sweet taste processing after recovery from anorexia and bulimia nervosa. Am J Psychiatry. 2013;170:1143-51.

78. Wagner A, Aizenstein H, Mazurkewicz L, Fudge J, Frank GK, Putnam K, et al. Altered insula response to taste stimuli in individuals recovered from restrictingtype anorexia nervosa. Neuropsychopharmacology. 2007;33:513-23.

79. Kodama N, Moriguchi $\mathrm{Y}$, Takeda $\mathrm{A}$, Maeda $\mathrm{M}$, Ando $\mathrm{T}$, Kikuchi $\mathrm{H}$, et al. Neural correlates of body comparison and weight estimation in weight-recovered anorexia nervosa: a functional magnetic resonance imaging study. Biopsychosoc Med. 2018;12:15

80. Baudinet J, Simic M, Griffiths H, Donnelly C, Stewart C, Goddard E. Targeting maladaptive overcontrol with radically open dialectical behaviour therapy in a day programme for adolescents with restrictive eating disorders: an uncontrolled case series. J Eat Disord. 2020;8:1-13.

81. Hempel R, Vanderbleek E, Lynch TR. Radically open DBT: targeting emotional loneliness in anorexia nervosa. Eat Disord. 2018;26:92-104. 
82. Wegner DM, Erber R. The hyperaccessibility of suppressed thoughts. J Pers Soc Psychol. 1992;63:903-12.

83. Wenzlaff RM, Wegner DM. Thought suppression. Annu Rev Psychol. 2000;51:59-91.

84. Doose A, King JA, Bernardoni F, Geisler D, Hellerhoff I, Weinert T, et al. Strengthened default mode network activation during delay discounting in adolescents with anorexia nervosa after partial weight restoration: a longitudinal fMRI study. J Clin Med. 2020;9:900.

85. Harrison A, Mountford VA, Tchanturia K. Social anhedonia and work and social functioning in the acute and recovered phases of eating disorders. Psychiatry Res. 2014;218:187-94.

86. Simon JJ, Stopyra MA, Friederich H-C. Neural processing of disorder-related stimuli in patients with anorexia nervosa: a narrative review of brain imaging studies. J Clin Med. 2019;8:1047.

87. Bang L, Treasure J, Rø $\varnothing$, Joos A. Advancing our understanding of the neurobiology of anorexia nervosa: translation into treatment. J Eat Disord. 2017;5:38.

88. Wierenga CE, Hill L, Knatz Peck S, McCray J, Greathouse L, Peterson D, et al. The acceptability, feasibility, and possible benefits of a neurobiologically-informed 5day multifamily treatment for adults with anorexia nervosa. Int J Eat Disord. 2018;51:863-9.

\section{ACKNOWLEDGEMENTS}

The authors would like to express their gratitude to all student assistants for their assistance with participant recruitment and data collection and thank all participants for their time and cooperation. We thank the Centre for Information Services and High-Performance Computing (ZIH) at TU Dresden for generous allocations of computer time. This work was supported by the Deutsche Forschungsgemeinschaft (EH 367/5-1 \& SFB 940) and the Swiss Anorexia Nervosa Foundation.

\section{AUTHOR CONTRIBUTIONS}

MS, HW, TG, and SE designed the study. MS, SP, SF, and S-MS recruited study participants. MS, SP, SF, JAK, S-MS, and DG collected the data. MS analyzed the data. SF, DG, and SE assisted in analyzing data. MS wrote the first draft of the manuscript. $\mathrm{SP}, \mathrm{SF}, \mathrm{JAK}, \mathrm{HW}, \mathrm{TG}$, and SE helped with literature search and editing. All authors contributed to writing and finalizing the article.

\section{FUNDING}

Open Access funding enabled and organized by Projekt DEAL.

\section{COMPETING INTERESTS}

The authors declare no competing interests.

\section{ADDITIONAL INFORMATION}

Supplementary information The online version contains supplementary material available at https://doi.org/10.1038/s41398-022-01797-1.

Correspondence and requests for materials should be addressed to Stefan Ehrlich.

Reprints and permission information is available at http://www.nature.com/ reprints

Publisher's note Springer Nature remains neutral with regard to jurisdictional claims in published maps and institutional affiliations.

(i) Open Access This article is licensed under a Creative Commons C. Attribution 4.0 International License, which permits use, sharing, adaptation, distribution and reproduction in any medium or format, as long as you give appropriate credit to the original author(s) and the source, provide a link to the Creative Commons license, and indicate if changes were made. The images or other third party material in this article are included in the article's Creative Commons license, unless indicated otherwise in a credit line to the material. If material is not included in the article's Creative Commons license and your intended use is not permitted by statutory regulation or exceeds the permitted use, you will need to obtain permission directly from the copyright holder. To view a copy of this license, visit http://creativecommons. org/licenses/by/4.0/.

(c) The Author(s) 2022 